\title{
MODE-INDEPENDENT $\mathcal{H}_{\infty}$ CONTROL OF DISCRETE-TIME MARKOVIAN JUMP LINEAR SYSTEMS *
}

\author{
Carlos E. de Souza* \\ * Department of Systems and Control \\ Laboratório Nacional de Computação Científica - LNCC \\ Av. Getulio Vargas 333, 25651-075 Petrópolis, RJ, Brazil \\ e-mail: csouza@lncc.br
}

\begin{abstract}
This paper addresses the problem of $\mathcal{H}_{\infty}$ control of discrete-time linear systems with Markovian jumping parameters. The main contribution of the paper is to provide a method for designing a state-feedback $\mathcal{H}_{\infty}$ control for systems where the jumping parameter is not accessible. The cases where the transition probability matrix of the Markov process is either exactly known, or unknown but belongs to a given polytope, are treated. The robust $\mathcal{H}_{\infty}$ control problem for systems with polytopic-type uncertain state-space model matrices is also considered and a state-feedback control design based on a Lyapunov function that depends on the uncertain parameters is developed. The proposed control designs are given in terms of linear matrix inequalities. Illustrative numerical examples are provided to demonstrate the effectiveness of the derived results. Copyright ${ }^{\circledR 2005 ~ I F A C . ~}$
\end{abstract}

Keywords: $\mathcal{H}_{\infty}$ control, Markov model, discrete-time systems, control synthesis, jump process.

\section{INTRODUCTION}

Over the past few years, Markovian jump linear (MJL) systems have been attracting an increasing attention. This class of systems is very appropriate to model plants whose structure is subject to random abrupt changes due to, for instance, random component failures, abrupt environment disturbance, changes of the operating point of a linearized model of a nonlinear system, etc. A number of control problems related to discretetime MJL systems has been analysed by several authors; see, for instance, Blair and Sworder (1975), Boukas and Liu (2001), Costa and do Val (1996), Costa and Fragoso (1993), Costa and Marques (2000), Fragoso (1989), Fragoso et al. (1995), Ji and Chizeck (1990), Ji et al. (1991), Morozan (1983), Shi et al. (1999), Wonham (1970)

\footnotetext{
* This work was supported in part by "Conselho Nacional de Desenvolvimento Científico e Tecnológico - CNPq", Brazil, under grants PRONEX 0331.00/00, 30.2317/023/PQ, 47.2920/03-0/APQ and IM-AGIMB.
}

and the references therein. In particular with regard to $\mathcal{H}_{\infty}$ control of discrete-time MJL systems, state-feedback control have been studied in, for instance, Costa and do Val (1996) and Fragoso et al. (1995), whereas robust $\mathcal{H}_{\infty}$ control schemes have been proposed in, e.g. Boukas and Liu (2001) and Shi et al. (1999). A common feature of the existing $\mathcal{H}_{\infty}$ control methods is that the jumping parameter is assumed to be accessible and is required for the implementation of the controller, which is a MJL system as well. To the best of the author's knowledge, to date the problem of state-feedback $\mathcal{H}_{\infty}$ control of discrete-time MJL systems with a non-accessible jumping parameter has not yet been fully addressed.

This paper addresses the problem of $\mathcal{H}_{\infty}$ control of discrete-time MJL systems where the jumping parameter is not accessible. Attention is focused on the design of a fixed static state-feedback controller, i.e. a mode-independent controller, that ensures mean square stability and a prescribed 
upper-bound on the $\mathcal{H}_{\infty}$ norm of the closed-loop system. Robust state-feedback $\mathcal{H}_{\infty}$ controllers are also derived for MJL systems subject to polytopictype parameter uncertainty in either the transition probability matrix of the Markov process, or in the matrices of the state-space model for the possible modes of operation of the system. In the later case, we propose a robust $\mathcal{H}_{\infty}$ control design based on a Lyapunov function that depends on the uncertain parameters. The control designs are given in terms of linear matrix inequalities (LMIs). The potentials of the proposed methods are demonstrated via two examples.

Notation. Throughout the paper the superscript " $T$ " stands for matrix transposition, $\mathbb{R}^{n}$ and $\mathbb{R}^{n \times m}$ denote the $n$-dimensional Euclidean space and the set of $n \times m$ real matrices, respectively, $\operatorname{diag}\{\cdots\}$ stands for a block-diagonal matrix, and for a symmetric block matrix, the symbol $\star$ denotes the transpose of the blocks outside the main diagonal block. For a real matrix $S, S>0(S<0)$ means that $S$ is symmetric and positive definite (negative definite) and $\ell_{2}$ denotes the space of squared summable vector sequences of a given dimension over the non-negative integers, with norm $\|x\|_{2}=\left[\sum_{k=0}^{\infty} x^{T}(k) x(k)\right]^{\frac{1}{2}}$.

\section{PROBLEM FORMULATION}

Fix an underlying probability space $(\Omega, \mathcal{F}, \mathbb{P})$ and consider the MJL system $(\mathcal{S})$ :

$$
\begin{aligned}
x(k+1) & =A\left(\theta_{k}\right) x(k)+J\left(\theta_{k}\right) u(k)+B\left(\theta_{k}\right) w(k) \\
z(k) & =C\left(\theta_{k}\right) x(k)+D\left(\theta_{k}\right) u(k)
\end{aligned}
$$

where $x(k) \in \mathbb{R}^{n}$ is the state, $u(k) \in \mathbb{R}^{n_{u}}$ is the control input, $w(k) \in \mathbb{R}^{n_{w}}$ is the disturbance input, which is assumed to be an arbitrary signal in $\ell_{2}$, and $z(k) \in \mathbb{R}^{n_{z}}$ is the controlled output. $\left\{\theta_{k}\right\}$ is a discrete-time homogeneous Markov chain with finite state-space $\Xi=\{1, \ldots, N\}$ and stationary transition probability matrix $\Lambda=\left[\lambda_{i j}\right]$, where

$$
\lambda_{i j}:=\mathbb{P}\left\{\theta_{k+1}=j \mid \theta_{k}=i\right\} .
$$

The set $\Xi$ comprises the operation modes of system $(\mathcal{S})$ and for each possible value of $\theta_{k}=i, i \in$ $\Xi$, we denote the matrices associated with the " $i$ th mode" by

$$
\begin{gathered}
A_{i}=A\left(\theta_{t}=i\right), \quad B_{i}=B\left(\theta_{t}=i\right), \quad C_{i}=C\left(\theta_{t}=i\right), \\
D_{i}=D\left(\theta_{t}=i\right), \quad J_{i}=J\left(\theta_{t}=i\right)
\end{gathered}
$$

where $A_{i}, B_{i}, C_{i}, D_{i}$ and $J_{i}$ are known real constant matrices for any $i \in \Xi$.

No assumption is made on the accessibility of the jumping process $\left\{\theta_{k}\right\}$. This is in contrast with existing approaches of $\mathcal{H}_{\infty}$ control of MJL systems, such as those of Costa and do Val (1996), Fragoso et al. (1995), and Shi et al. (1999), which require the jumping parameter to be accessible.
Before formulating the $\mathcal{H}_{\infty}$ control problem, in the sequel we present the notion of internal mean square stability and a related result.

Definition 1. System $(\mathcal{S})$ is said to be internally mean square stable (IMSS), if the solution to the stochastic difference equation:

$$
x(k+1)=A\left(\theta_{k}\right) x(k)
$$

is such that $E\left[\|x(k)\|^{2}\right] \rightarrow 0$, as $k \rightarrow \infty$ for any finite initial condition $x(0) \in \mathbb{R}^{n}$ and $\theta_{0} \in \Xi$.

Lemma 1. (Costa and Fragoso, 1993) System $(\mathcal{S})$ is IMSS if and only if there exist matrices $P_{i}>0, i=1, \ldots, N$, satisfying the following inequalities:

$$
P_{i}-A_{i}^{T} \sum_{j=1}^{N} \lambda_{i j} P_{j} A_{i}>0, \quad i=1, \ldots, N .
$$

Next, we recall the notion of $\mathcal{H}_{\infty}$ norm and a version of the bounded real lemma (BRL) for MJL systems. To this end, let the MJL system:

$$
\begin{aligned}
\left(\mathcal{S}_{1}\right): x(k+1) & =A\left(\theta_{k}\right) x(k)+B\left(\theta_{k}\right) w(k) \\
z(k) & =C\left(\theta_{k}\right) x(k)
\end{aligned}
$$

where $x(k) \in \mathbb{R}^{n}, w(k) \in \mathbb{R}^{n_{w}}$ and $z(k) \in \mathbb{R}^{n_{z}}$, whereas $\theta_{k}$ and the matrices $A\left(\theta_{k}\right), B\left(\theta_{k}\right)$ and $C\left(\theta_{k}\right)$ are as in system $(\mathcal{S})$.

Definition 2. Let the system $\left(\mathcal{S}_{1}\right)$ be IMSS. The $\mathcal{H}_{\infty}$ norm of $\left(\mathcal{S}_{1}\right)$, i.e. the $\ell_{2}$-induced gain, is defined by

$$
\left\|\mathcal{S}_{1}\right\|_{\infty}:=\sup _{w \in \ell_{2}}\left\{\frac{\|z\|_{2 s}}{\|w\|_{2}} ; w \not \equiv 0, x(0)=0\right\}
$$

where

$$
\|z\|_{2 s}=\left\{\mathbf{E}\left[\sum_{k=0}^{\infty} z^{T}(k) z(k)\right]\right\}^{\frac{1}{2}} .
$$

Lemma 2. (Costa and do Val, 1996) Given the system $\left(\mathcal{S}_{1}\right)$ and a scalar $\gamma>0$, the following conditions are equivalent:

(a) System $\left(\mathcal{S}_{1}\right)$ is IMSS and $\left\|\mathcal{S}_{1}\right\|_{\infty}<\gamma$;

(b) There exist matrices $P_{i}>0, i=1, \ldots, N$, satisfying the following LMIs:

$$
\left[\begin{array}{cccc}
\bar{P}_{i} & \bar{P}_{i} A_{i} & 0 & \bar{P}_{i} B_{i} \\
A_{i}^{T} \bar{P}_{i} & P_{i} & C_{i}^{T} & 0 \\
0 & C_{i} & \gamma I & 0 \\
B_{i}^{T} \bar{P}_{i} & 0 & 0 & \gamma I
\end{array}\right]>0, i=1, \ldots, N
$$

where

$$
\bar{P}_{i}=\sum_{j=1}^{N} \lambda_{i j} P_{j}, i=1, \ldots, N .
$$

Moreover, $V\left(x(k), \theta_{k}\right)=x^{T}(k) P\left(\theta_{k}\right) x(k)$, where $P\left(\theta_{k}\right)=P_{i}$ for $\theta_{k}=i, i=1, \ldots, N$ is a Lyapunov function for the unforced system of $\left(\mathcal{S}_{1}\right)$. 
A version of the BRL for MJL systems which is suitable for control design in terms of LMIs is presented below.

Lemma 3. Given the system $\left(\mathcal{S}_{1}\right)$ and a scalar $\gamma>0$, the following conditions are equivalent:

(a) System $\left(\mathcal{S}_{1}\right)$ is IMSS and $\left\|\mathcal{S}_{1}\right\|_{\infty}<\gamma$,

(b) There exist matrices $X_{i}>0, i=1, \ldots, N$, satisfying the following LMIs:

$$
\left[\begin{array}{cccc}
\mathcal{X} & \Lambda_{i}^{T} A_{i} X_{i} & 0 & \Lambda_{i}^{T} B_{i} \\
X_{i} A_{i}^{T} \Lambda_{i} & X_{i} & X_{i} C_{i}^{T} & 0 \\
0 & C_{i} X_{i} & \gamma I & 0 \\
B_{i}^{T} \Lambda_{i} & 0 & 0 & \gamma I
\end{array}\right]>0, i=1, \ldots, N
$$

where

$$
\begin{aligned}
\mathcal{X} & =\operatorname{diag}\left\{X_{1}, \cdots, X_{N}\right\} \\
\Lambda_{i} & =\left[\sqrt{\lambda_{i 1}} I \cdots \sqrt{\lambda_{i N}} I\right] .
\end{aligned}
$$

Moreover, $V\left(x(k), \theta_{k}\right)=x^{T}(k) P\left(\theta_{k}\right) x(k)$, where $P\left(\theta_{k}\right)=X_{i}^{-1}$ for $\theta_{k}=i, i=1, \ldots, N$ is a Lyapunov function for the unforced system of $\left(\mathcal{S}_{1}\right)$.

Proof. By Schur's complement, (5) can be rewritten as

$$
\left[\begin{array}{cccc}
\mathcal{P}^{-1} & \Lambda_{i}^{T} A_{i} & 0 & \Lambda_{i}^{T} B_{i} \\
A_{i}^{T} \Lambda_{i} & P_{i} & C_{i}^{T} & 0 \\
0 & C_{i} & \gamma I & 0 \\
B_{i}^{T} \Lambda_{i} & 0 & 0 & \gamma I
\end{array}\right]>0, i=1, \ldots, N
$$

where

$$
\mathcal{P}=\operatorname{diag}\left\{P_{1}, \cdots, P_{N}\right\} .
$$

Then, pre- and post-multiplying the above inequality by $\operatorname{diag}\left\{I, P_{i}^{-1}, I, I\right\}$ and its transpose, respectively, and denoting $X_{i}=P_{i}^{-1}$, it follows that the LMIs of (5) and (6) are equivalent. $\nabla \nabla \nabla$

It should be remarked that in the case where there are no jumps in system $\left(\mathcal{S}_{1}\right)$, Lemmas 2 and 3 reduce to a well known BRL for discrete-time linear systems.

This paper is concerned with the following $\mathcal{H}_{\infty}$ control problem for the system $(\mathcal{S})$ :

Given the system $(\mathcal{S})$ and a scalar $\gamma>0$, find a mode-independent control law

$$
u(k)=K x(k)
$$

such that the resulting closed-loop system

$$
\begin{aligned}
\left(\mathcal{S}_{c}\right): x(k+1) & =A_{c}\left(\theta_{k}\right) x(k)+B\left(\theta_{k}\right) w(k) \\
z(k) & =C_{c}\left(\theta_{k}\right) x(k)
\end{aligned}
$$

where

$$
\begin{aligned}
& A_{c}\left(\theta_{k}\right)=A\left(\theta_{k}\right)+J\left(\theta_{k}\right) K \\
& C_{c}\left(\theta_{k}\right)=C\left(\theta_{k}\right)+D\left(\theta_{k}\right) K
\end{aligned}
$$

is $I M S S$ and $\left\|\mathcal{S}_{c}\right\|_{\infty}<\gamma$.

\section{3. $\mathcal{H}_{\infty}$ CONTROL}

First, we present an alternative version of the BRL for MJL systems. This new $\mathcal{H}_{\infty}$ norm characterization will be fundamental in the derivation of the mode-independent $\mathcal{H}_{\infty}$ control methods proposed in this paper.

Theorem 1. Given the system $\left(\mathcal{S}_{1}\right)$ and a scalar $\gamma>0$, the following conditions are equivalent:

(a) System $\left(\mathcal{S}_{1}\right)$ is IMSS and $\left\|\mathcal{S}_{1}\right\|_{\infty}<\gamma$,

(b) There exist matrices $X_{i}$ and $G_{i}, i=1, \ldots, N$ satisfying the following LMIs:

$$
\begin{array}{cccc}
{\left[\begin{array}{cccc}
\mathcal{X} & \Lambda_{i}^{T} A_{i} G_{i} & 0 & \Lambda_{i}^{T} B_{i} \\
G_{i}^{T} A_{i}^{T} \Lambda_{i} & G_{i}+G_{i}^{T}-X_{i} & G_{i}^{T} C_{i}^{T} & 0 \\
0 & C_{i} G_{i} & \gamma I & 0 \\
B_{i}^{T} \Lambda_{i} & 0 & 0 & \gamma I
\end{array}\right]>0} \\
\multicolumn{2}{c}{i=1, \ldots, N}
\end{array}
$$

where the matrices $\mathcal{X}$ and $\Lambda_{i}$ are as in (7) and (8), respectively. Moreover, $V\left(x(k), \theta_{k}\right)=$ $x^{T}(k) P\left(\theta_{k}\right) x(k)$, where $P\left(\theta_{k}\right)=X_{i}^{-1}$ when $\theta_{k}=$ $i, i=1, \ldots, N$ is a Lyapunov function for the unforced system of $\left(\mathcal{S}_{1}\right)$.

Proof. Necessity: By Lemma 3, there exist matrices $X_{i}>0, i=1, \ldots, N$ satisfying (6). Then, it follows immediately that (12) holds with the same matrices $X_{i}$ and with $G_{i}=X_{i}, i=1, \ldots, N$.

Sufficiency: Pre- and post-multiplying (12) by

$$
\left[\begin{array}{cccc}
I & -\Lambda_{i}^{T} A_{i} & 0 & 0 \\
0 & -C_{i} & I & 0 \\
0 & 0 & 0 & I
\end{array}\right]
$$

and its transpose, respectively leads to

$$
\left[\begin{array}{ccc}
\mathcal{X}-\Lambda_{i}^{T} A_{i} X_{i} A_{i}^{T} \Lambda_{i} & -\Lambda_{i}^{T} A_{i} X_{i} C_{i}^{T} & \Lambda_{i}^{T} B_{i} \\
-C_{i} X_{i} A_{i}^{T} \Lambda_{i} & \gamma I-C_{i} X_{i} C_{i}^{T} & 0 \\
B_{i}^{T} \Lambda_{i} & 0 & \gamma I
\end{array}\right]>0 .
$$

Then, the result follows immediately by applying Schur's complement to the above inequality and considering Lemma 3 .

$\nabla \nabla \nabla$

Theorem 1 is equivalent to Lemma 3 and thus has no advantages over that lemma when used as a necessary and sufficient condition for ensuring an upper-bound on $\left\|\mathcal{S}_{1}\right\|_{\infty}$. However, due to the extra degrees of freedom introduced by the variables $G_{i}$, Theorem 1 offers potential advantages over Lemma 3 when constraints are imposed to the matrices $G_{i}$, including allowing for the design of a mode-independent state-feedback $\mathcal{H}_{\infty}$ control law. As it will be established in the next theorem, this can be achieved by imposing the constraints $G_{i}=G, i=1, \ldots, N$. Note that in this situation the feasibility of (12), applied to closed-loop system $\left(\mathcal{S}_{c}\right)$, is only sufficient for $\left(\mathcal{S}_{c}\right)$ to be IMSS and $\left\|\mathcal{S}_{c}\right\|_{\infty}<\gamma$. 
The next theorem presents the mode-independent state-feedback $\mathcal{H}_{\infty}$ control design.

Theorem 2. Consider the system $(\mathcal{S})$ and let $\gamma>$ 0 be a given scalar. Suppose that there exist matrices $G, Y$ and $X_{i}, i=1, \ldots, N$ satisfying the following LMIs:

$$
\left[\begin{array}{cccc}
\mathcal{X} & \star & \star & \star \\
\Upsilon_{i}(G, Y)^{T} \Lambda_{i} & G+G^{T}-X_{i} & \star & \star \\
0 & \Omega_{i}(G, Y) & \gamma I & \star \\
B_{i}^{T} \Lambda_{i} & 0 & 0 & \gamma I
\end{array}\right]>0,
$$

where the matrices $\mathcal{X}$ and $\Lambda_{i}$ are as in (7) and (8), respectively, and

$\Upsilon_{i}(G, Y)=A_{i} G+J_{i} Y, \quad \Omega_{i}(G, Y)=C_{i} G+D_{i} Y$

Then, the mode-independent control law

$$
u(k)=K x(k), \quad K=Y G^{-1}
$$

ensures that the resulting closed-loop system $\left(\mathcal{S}_{c}\right)$ is IMSS and $\left\|\mathcal{S}_{c}\right\|_{\infty}<\gamma$.

Proof. First, note that (13) implies that the matrix $G$ is nonsingular. Applying Theorem 1 to the closed-loop system $\left(\mathcal{S}_{c}\right)$ and setting $G_{i}=$ $G, i=1, \ldots, N$, it follows that the system $\left(\mathcal{S}_{c}\right)$ is IMSS and $\left\|\mathcal{S}_{c}\right\|_{\infty}<\gamma$ if there exist matrices $G, K$ and $X_{i}, i=1, \ldots, N$ such that:

$$
\left[\begin{array}{cccc}
\mathcal{X} & \Lambda_{i}^{T} A_{c_{i}} G & 0 & \Lambda_{i}^{T} B_{i} \\
G^{T} A_{c_{i}}^{T} \Lambda_{i} & G+G^{T}-X_{i} & G^{T} C_{c_{i}}^{T} & 0 \\
0 & C_{c_{i}} G & \gamma I & 0 \\
B_{i}^{T} \Lambda_{i} & 0 & 0 & \gamma I
\end{array}\right]>0
$$

where

$$
A_{c_{i}}=A_{i}+J_{i} K, \quad C_{c_{i}}=C_{i}+D_{i} K .
$$

Then, by setting $Y=K G$ it results that (16) becomes identical to the LMIs of (13) and the controller gain of (15) follows immediately. $\nabla \nabla \nabla$

Observe that the problem of finding the modeindependent control law of Theorem 2 with a minimized $\gamma$ can be solved via a convex optimization problem.

Remark 1. In the case of one mode operation, i.e. when there are no jumps in system $(\mathcal{S})$, Theorem 2 reduces to a well known state-feedback $\mathcal{H}_{\infty}$ control result for discrete-time linear systems (Boyd et al., 1994), which is indeed necessary and sufficient for the problem solvability.

Remark 2. It should be noted that when the jumping parameter is accessible, Theorem 2 can be extended to allow for the design of a Markovian jump control law, namely, a mode-dependent control law of the form:

$$
u(k)=K\left(\theta_{k}\right) x(k) .
$$

Indeed, it follows from the proof of Theorem 2 that this problem can be solved in terms of the feasibility of the LMIs of (13) with $G$ and $Y$ replaced by $G_{i}$ and $Y_{i}$, respectively. In this case, a suitable gain matrix $K\left(\theta_{k}\right)$ is given by:

$$
K\left(\theta_{k}\right)=Y_{i} G_{i}^{-1}, \text { when } \theta_{k}=i .
$$

Note that in this situation, Theorem 2 provides necessary and sufficient conditions for the solution of this $\mathcal{H}_{\infty}$ control problem and this theorem is equivalent to the main result of Costa and do Val (1996), when restricted to the case where the Markov chain takes value in a finite set.

\section{4. $\operatorname{ROBUST} \mathcal{H}_{\infty}$ CONTROL}

Theorem 2 can be easily extended to the case where the transition probability matrix $\Lambda=\left[\lambda_{i j}\right]$ is unknown, but belongs to a given polytope, namely $\Lambda \in \Pi_{\Lambda}$, where $\Pi_{\Lambda}$ is a polytope with vertices $\Lambda_{i}, i=1, \ldots, v$, i.e.

$$
\Pi_{\Lambda}:=\left\{\Lambda \mid \Lambda=\sum_{r=1}^{v} \alpha_{r} \Lambda_{r} ; \quad \alpha_{r} \geq 0, \sum_{r=1}^{v} \alpha_{r}=1\right\}
$$

where $\Lambda_{r}=\left[\lambda_{i j}^{(r)}\right], i, j=1, \ldots, N, r=1, \ldots, v$ are given transition probability matrices. Note that the convex hull of transition probability matrices is also a transition probability matrix.

Attention is focused on designing a mode independent control law (9) that ensures internal mean square stability and a prescribed $\mathcal{H}_{\infty}$ performance of the close-loop system $\left(\mathcal{S}_{c}\right)$ over the polytope $\Pi_{\Lambda}$. In the above setting, and considering that the inequalities of (13) are affine in $\lambda_{i j}$, the following robust state-feedback $\mathcal{H}_{\infty}$ control result follows readily from Theorem 2 .

Theorem 3. Consider the system $(\mathcal{S})$ with an uncertain transition probability matrix $\Lambda$ belonging to the polytope $\Pi_{\Lambda}$ and let $\gamma>0$ be a given scalar. Suppose that there exist matrices $G, Y$ and $X_{i}, i=1, \ldots, N$ satisfying the following LMIs:

$$
\begin{gathered}
{\left[\begin{array}{cccc}
\mathcal{X} & \star & \star & \star \\
\Upsilon_{i}(G, Y)^{T} \Lambda_{i}^{(r)} & G+G^{T}-X_{i} & \star & \star \\
0 & \Omega_{i}(G, Y) & \gamma I & \star \\
B_{i}^{T} \Lambda_{i}^{(r)} & 0 & 0 & \gamma I
\end{array}\right]>0} \\
\quad r=1, \ldots, v, \quad i=1, \ldots, N
\end{gathered}
$$

where

$$
\Lambda_{i}^{(r)}=\left[\sqrt{\lambda_{i 1}^{(r)}} I \cdots \sqrt{\lambda_{i N}^{(r)}} I\right]
$$

and the other matrices are the same as in Theorem 2. Then, the mode-independent control law

$$
u(k)=K x(k), \quad K=Y G^{-1}
$$

ensures that the resulting closed-loop system $\left(\mathcal{S}_{c}\right)$ is IMSS and $\left\|\mathcal{S}_{c}\right\|_{\infty}<\gamma$ over the polytope $\Pi_{\Lambda} . \square$ 
In the sequel, we shall consider the design of a robust state-feedback $\mathcal{H}_{\infty}$ controller in the case where the matrices $A_{i}, B_{i}, C_{i}, D_{i}$ and $J_{i}, i=$ $1, \ldots, N$, of system $(\mathcal{S})$ are uncertain, but belong to given matrix polytopes $\Pi_{i}, i=1, \ldots, N$, described by:

$$
\begin{aligned}
& \Pi_{i}:=\left\{\left(A_{i}, B_{i}, C_{i}, D_{i}, J_{i}\right) \mid\left(A_{i}, B_{i}, C_{i}, D_{i}, J_{i}\right)\right. \\
= & \left.\sum_{r=1}^{v_{i}} \alpha_{i r}\left(A_{i r}, B_{i r}, C_{i r}, D_{i r}, J_{i r}\right) ; \alpha_{i r} \geq 0, \sum_{r=1}^{v_{i}} \alpha_{i r}=1\right\}
\end{aligned}
$$

where $v_{i}$ is the number of vertices of $\Pi_{i}$ and $A_{i r}$, $B_{i r}, C_{i r}, D_{i r}$ and $J_{i r}$ are given matrices.

The control law (9) to be designed should guarantee internal mean square stability and a prescribed $\mathcal{H}_{\infty}$ performance for the closed-loop system $\left(\mathcal{S}_{c}\right)$ over the polytopes $\Pi_{i}, i=1, \ldots, N$. To solve this robust $\mathcal{H}_{\infty}$ control problem, a parametric Lyapunov function approach is adopted, where the Lyapunov function depends on the uncertain parameter vectors $\alpha_{i}=\left[\alpha_{i 1}, \ldots, \alpha_{i v_{i}}\right]^{T}$ that characterizes the uncertainty matrix polytopes $\Pi_{i}$. Specifically, denoting $\alpha=\left(\alpha_{1}, \ldots, \alpha_{N}\right)$, the Lyapunov function is of the form:

with

$$
V\left(x(k), \theta_{k}, \alpha\right)=x^{T}(k) P\left(\theta_{k}, \alpha\right) x(k),
$$

$$
\begin{gathered}
P\left(\theta_{k}, \alpha\right)=X_{i}^{-1}\left(\alpha_{i}\right), \text { for } \theta_{k}=i, i=1, \ldots, N \\
X_{i}\left(\alpha_{i}\right)=\sum_{r=1}^{v_{i}} \alpha_{i r} X_{i}^{(r)}
\end{gathered}
$$

where $X_{i}^{(r)}, i=1, \ldots, N, r=1, \ldots, v_{i}$ are symmetric positive definite matrices and $\alpha_{i r}$ are the non-negative scalars of (21).

As the LMIs of (13) are affine in the matrices $A_{i}, B_{i}, C_{i}, D_{i}$ and $J_{i}$, the following robust $\mathcal{H}_{\infty}$ control result is obtained from Theorem 2 .

Theorem 4. Consider the system $(\mathcal{S})$ with the matrices $A_{i}, B_{i}, C_{i}, D_{i}$ and $J_{i}$ belonging to the polytope $\Pi_{i}, i=1, \ldots, N$ and let $\gamma>0$ be a given scalar. Suppose that there exist matrices $G, Y$ and $X_{i}^{(r)}, i=1, \ldots, N, r=1, \ldots, v_{i}$, satisfying the following LMIs:

$$
\begin{gathered}
{\left[\begin{array}{cccc}
\mathcal{X}^{\left[\pi_{i r}\right]} & \star & \star & \star \\
\Upsilon_{i r}(G, Y)^{T} \Lambda_{i} & G+G^{T}-X_{i}^{(r)} & \star & \star \\
0 & \Omega_{i r}(G, Y) & \gamma I & \star \\
B_{i r}^{T} \Lambda_{i} & 0 & 0 & \gamma I
\end{array}\right]>0} \\
\forall \pi_{i r} \in \mathcal{I}_{i r}, r=1, \ldots, v_{i}, i=1, \ldots, N
\end{gathered}
$$

where the matrix $\Lambda_{i}$ is as defined in (8),

$$
\begin{gathered}
\Upsilon_{i r}(G, Y)=A_{i r} G+J_{i r} Y \\
\Omega_{i r}(G, Y)=C_{i r} G+D_{i r} Y
\end{gathered}
$$

and $\mathcal{I}_{\text {ir }}$ denotes the set of all $N$-tuples

$$
\pi_{i r}=\left[p_{1}, \ldots p_{i-1}, r, p_{i+1}, \ldots, p_{N}\right],
$$

$$
\forall p_{s} \in\left\{1, \ldots, v_{s}\right\}, s=1, \ldots, N, s \neq i
$$

where to each $\pi_{i r}$ is associated the matrix

$\mathcal{X}^{\left[\pi_{i r}\right]}=\operatorname{diag}\left\{X_{1}^{\left(p_{1}\right)}, \cdots, X_{i-1}^{\left(p_{i-1}\right)}, X_{i}^{(r)}, X_{i+1}^{\left(p_{i+1}\right)}, \cdots, X_{N}^{\left(p_{N}\right)}\right\}$

Then, the mode-independent control law

$$
u(k)=K x(k), \quad K=Y G^{-1}
$$

ensures that the resulting closed-loop system $\left(\mathcal{S}_{c}\right)$ is IMSS and $\left\|\mathcal{S}_{c}\right\|_{\infty}<\gamma$ over the polytopes $\Pi_{i}, i=$ $1, \ldots, N$.

It should be remarked that the robust $\mathcal{H}_{\infty}$ control design method of Theorem 4 is based on a Lyapunov function that depends on both the jumping parameter $\theta_{k}$ and the uncertain parameters in the system matrices for each operation mode. This is in contrast with existing robust $\mathcal{H}_{\infty}$ control results for MJL systems, such as those of Boukas and Liu (2001) and Shi et al. (1999), which are based on Lyapunov functions that are independent of the system uncertain parameters and require the jumping parameter to be accessible.

Theorem 4 reduces to a control method based on an uncertainty-independent Lyapunov function by imposing the constraints $X_{i}^{(r)}=X_{i}, r=1, \ldots, v_{i}$. Similarly to the case of systems without jumps, it turns out that the result of Theorem 4 is less conservative than those based on an uncertaintyindependent Lyapunov function.

Remark 3. Observe that Remark 2 also applies to Theorems 3 and 4 . In particular, a modedependent robust $\mathcal{H}_{\infty}$ control method based on an uncertainty-dependent Lyapunov function is readily obtained from Theorem 4 by replacing the matrices $G$ and $Y$ in $(22)$ by $G_{i}$ and $Y_{i}$, respectively. In addition, Theorem 4 with $G_{i}$ in lieu of $G$, and $B_{i r}=0, C_{i r}=0, D_{i r}=0$ and $J_{i r}=0$ leads to a new robust mean square stability condition for MJL systems based on an uncertainty-dependent Lyapunov function. To the best of the authors knowledge, such robust stability and $\mathcal{H}_{\infty}$ control results are the first ones in the context of MJL systems.

\section{EXAMPLES}

Two examples are presented to illustrate the applicability of Theorems 2-4.

Example 1: Consider the example in Boukas and Liu (2001) without time-delay and uncertainty, namely, let the system $(\mathcal{S})$ with three operating modes described by:

$A_{1}=\left[\begin{array}{cc}1 & 0 \\ 0 & 1.2\end{array}\right], A_{2}=\left[\begin{array}{cc}1.13 & 0 \\ 0.16 & 0.478\end{array}\right], A_{3}=\left[\begin{array}{cc}0.3 & 0.13 \\ 0.16 & 1.14\end{array}\right]$,

$B_{1}=\left[\begin{array}{cc}0.1 & 0 \\ 0.1 & -0.1\end{array}\right], B_{2}=\left[\begin{array}{cc}-0.1 & 0.1 \\ 0 & 0.1\end{array}\right], B_{3}=\left[\begin{array}{cc}0.1 & 0 \\ 0 & 0\end{array}\right]$, 


$$
\begin{gathered}
C_{1}=\left[\begin{array}{ll}
0.1 & 0 \\
0.1 & 0
\end{array}\right], C_{2}=\left[\begin{array}{cc}
0 & 0.1 \\
0.1 & 0
\end{array}\right], C_{3}=\left[\begin{array}{cc}
0.4 & -0.1 \\
0.1 & 0
\end{array}\right], \\
D_{1}=\left[\begin{array}{rr}
0.2 & 0.2 \\
-0.2 & 0.1
\end{array}\right], D_{2}=\left[\begin{array}{ll}
0.1 & 0.1 \\
0.2 & 0.1
\end{array}\right], D_{3}=\left[\begin{array}{cc}
0 & 0.1 \\
-0.2 & 0.1
\end{array}\right], \\
J_{1}=\left[\begin{array}{ll}
0.1 & 0 \\
0.1 & 0
\end{array}\right], J_{2}=\left[\begin{array}{cc}
0.2 & 0.1 \\
0 & -0.1
\end{array}\right], J_{3}=\left[\begin{array}{rr}
0 & 0.1 \\
0 & -0.1
\end{array}\right] .
\end{gathered}
$$

Regarding the transition probability matrix $\Lambda$, two cases will be considered:

Case 1: $\Lambda$ is exactly known and given by

$$
\Lambda=\left[\begin{array}{ccc}
0.2 & 0.8 & 0 \\
0 & 0.5 & 0.5 \\
0 & 0.3 & 0.7
\end{array}\right]
$$

The minimum $\gamma$ obtained with Theorem 2 is 0.39881 and the state-feedback gain is

$$
K=\left[\begin{array}{rr}
-1.8621 & -1.5790 \\
0.9566 & 2.5834
\end{array}\right]
$$

It should be noted that the actual $\mathcal{H}_{\infty}$ norm of the resulting closed-loop system is 0.32734 .

Case 2: $\Lambda$ is unknown, but belongs to a polytope $\Pi_{\Lambda}$, as defined in (19), with 2 vertices given by:

$$
\Lambda_{1}=\left[\begin{array}{ccc}
0.15 & 0.85 & 0 \\
0 & 0.45 & 0.55 \\
0 & 0.2 & 0.8
\end{array}\right], \quad \Lambda_{2}=\left[\begin{array}{ccc}
0.25 & 0.75 & 0 \\
0 & 0.6 & 0.4 \\
0 & 0.4 & 0.6
\end{array}\right] \text {. }
$$

The minimum $\gamma$ achieved with the robust $\mathcal{H}_{\infty}$ control design of Theorem 3 is 0.43900 and the corresponding controller gain is

$$
K=\left[\begin{array}{rr}
-2.0500 & -2.6286 \\
1.3421 & 3.5567
\end{array}\right]
$$

Example 2: This example deals with the robust stability analysis of an uncertain MJL system with 2 operating modes described by:

$$
\Lambda=\left[\begin{array}{cc}
0.1 & 0.9 \\
0.15 & 0.85
\end{array}\right], \quad A_{1}=\left[\begin{array}{rcc}
1.1 & 0.6 & 0.2 \\
-0.3 & 0.9 & 0 \\
0.1 & 0 & 0.8
\end{array}\right],
$$

whereas $A_{2}$ is uncertain but belongs to a polytope with 2 vertices given by:

$$
A_{21}=\left[\begin{array}{ccc}
1 & 0.5 & 0 \\
-0.3 & 0.8 & 0 \\
0 & 0 & 0.4
\end{array}\right], \quad A_{22}=\left[\begin{array}{ccc}
1 & 0.4 & 0.01 \\
-0.1 & 0.8 & 0 \\
0 & 0 & 0.5
\end{array}\right]
$$

By the robust stability condition of Theorem 4 as described in Remark 3, the above system is robustly IMSS. However, if the Lyapunov function is constrained to be uncertainty-independent, i.e. by setting $X_{i}^{(r)}=X_{i}, r=1,2$, no conclusion can be drawn regarding the robust mean square stability of the above system. This result demonstrates the superiority of the uncertainty-dependent Lyapunov function approach.

\section{CONCLUSIONS}

This paper has addressed the problem of $\mathcal{H}_{\infty}$ control of discrete-time MJL systems with a nonaccessible jumping parameter. An LMI method is proposed for designing a mode-independent statefeedback control law that ensures mean square stability and a prescribed upper-bound on the $\mathcal{H}_{\infty}$ norm of the closed-loop system. Robust $\mathcal{H}_{\infty}$ control laws have also been proposed for MJL systems subject to polytopic-type parameter uncertainty in either the transition probability matrix of the Markov process, or in the matrices of the statespace model for the possible modes of operation of the system. In the later case, the control design is based on a Lyapunov function that depends on the uncertain parameters. The proposed $\mathcal{H}_{\infty}$ control design methods can be readily adapted to the case where the jumping parameter is accessible and a Markovian jump (mode-dependent) state-feedback control law is sought. A new robust mean square stability condition for discrete-time uncertain MJL systems has also been developed.

\section{REFERENCES}

Blair, W.P. and D.D. Sworder (1975). Feedback control of a class of linear discrete systems with jump parameters and quadratic cost criteria. Int. J. Control, 21, 833841.

Boukas, E.K. and Z.K. Liu (2001). Robust $\mathcal{H}_{\infty}$ control of a class of linear discrete-time Markovian jump linear systems with mode-dependent time-delays. IEEE Trans. Auotom. Control, 46, 1918-1924.

Boyd, S., L. El Ghaoui, E. Feron and V. Balakrishnan (1994). Linear Matrix Inequalities in System and Control Theory. SIAM, Philadelphia, PA.

Costa, O.L.V. and J.B.R. do Val (1996). Full information $\mathcal{H}_{\infty}$ control for discrete-time infinite Markov jump parameter systems. J. Math. Anal. Appl. 202, 578603.

Costa, O.L.V. and M.D. Fragoso (1993). Stability results for discrete-time linear systems with Markovian jump parameters. J. Math. Anal. Appl., 179, 154-178.

Costa, O.L.V. and R.P. Marques (2000). Robust $\mathcal{H}_{2}$ control for control for discrete-time Markovian jump linear systems. Int. J. Control, 73, 11-21.

Fragoso, M.D. (1995). Discrete-time jump LQG problem. Int. J. Systems Sci., 20, 2539-2545.

Fragoso, M.D., J.B.R. do Val and D.L. Pinto Jr (1995). Jump linear $\mathcal{H}_{\infty}$ control: The discrete-time case. Control Theory Adv. Tech., 10, 1459-1474.

Ji, Y. and H.J. Chizeck (1990). Jump linear quadratic gaussian control: Steady-state and testable conditions. Control Theory Adv. Tech., 6, 289-319.

Ji, Y., H.J. Chizeck, X. Feng and K.A. Loparo (1991). Stability and control of discrete-time jump linear systems. Control Theory Adv. Tech., 7, 247-270.

Morozan, T. (1983). Stabilization of some stochastic discrete-time control systems. Stochastic. Anal. Appl., 1, 89-116.

Shi, P., E.K. Boukas and R.K. Agarwal (1999). Robust control for Markovian jumping discrete-time systems. Int. J. Systems Sci., 30, pp. 787-797.

Wonham, W.H. (1970). Random differential equations in control theory. In: Probability Methods in Applied Mathematics (A.T. Bharucha-Reid, Ed.), 2, 131-212. Academic Press, New York. 